\title{
The Development of Brain Systems Associated with Successful Memory Retrieval of Scenes
}

\author{
Noa Ofen, ${ }^{1,3,4}$ Xiaoqian J. Chai, ${ }^{1,3}$ Karen D. I. Schuil, ${ }^{1,3,5}$ Susan Whitfield-Gabrieli, ${ }^{1,3}$ and John D. E. Gabrieli ${ }^{1,2,3}$ \\ ${ }^{1}$ Department of Brain and Cognitive Sciences, ${ }^{2}$ Harvard-MIT Division of Health Sciences and Technology, and ${ }^{3}$ McGovern Institute for Brain Research, \\ Massachusetts Institute of Technology, Cambridge, Massachusetts, 02139, ${ }^{4}$ Institute of Gerontology and Department of Pediatrics, Wayne State University, \\ Detroit, Michigan 48202, and 5Institute of Psychology, Erasmus University Rotterdam, 3000 DR Rotterdam, The Netherlands
}

Neuroanatomical and psychological evidence suggests prolonged maturation of declarative memory systems in the human brain from childhood into young adulthood. Here, we examine functional brain development during successful memory retrieval of scenes in children, adolescents, and young adults ages 8-21 via functional magnetic resonance imaging. Recognition memory improved with age, specifically for accurate identification of studied scenes (hits). Successful retrieval (correct old-new decisions for studied vs unstudied scenes) was associated with activations in frontal, parietal, and medial temporal lobe (MTL) regions. Activations associated with successful retrieval increased with age in left parietal cortex (BA7), bilateral prefrontal, and bilateral caudate regions. In contrast, activations associated with successful retrieval did not change with age in the MTL. Psychophysiological interaction analysis revealed that there were, however, age-relate changes in differential connectivity for successful retrieval between MTL and prefrontal regions. These results suggest that neocortical regions related to attentional or strategic control show the greatest developmental changes for memory retrieval of scenes. Furthermore, these results suggest that functional interactions between MTL and prefrontal regions during memory retrieval also develop into young adulthood. The developmental increase of memory-related activations in frontal and parietal regions for retrieval of scenes and the absence of such an increase in MTL regions parallels what has been observed for memory encoding of scenes.

\section{Introduction}

Declarative or explicit memory is comprised of three successive stages: (1) encoding, (2) storage or consolidation, and (3) retrieval. Neuroimaging studies have highlighted the involvement of medial temporal lobe (MTL), prefrontal, and parietal regions in memory retrieval in adults (Fletcher et al., 1998; Buckner and Wheeler, 2001; Kahn et al., 2004; Wagner et al., 2005; Konishi et al., 2000; Spaniol et al., 2009), but little is known about the development of the functional neural correlates for the retrieval of declarative memories. Structurally, there is prolonged anatomic maturation in prefrontal and parietal regions (Giedd et al., 1999; Sowell et al., 2003, 2004; Gogtay et al., 2004, 2006). Behavioral evidence suggests that retrieval strategies continue to mature through adolescence (Bauer, 2008). This study investigated the maturation of the neural correlates of memory retrieval from childhood through adolescence and into young adulthood.

There is growing evidence about the development of memory systems in the human brain and the different maturational tra-

\footnotetext{
Received March 1, 2011; revised May 16, 2012; accepted May 24, 2012.

Author contributions: N.O., X.J.C., and J.D.E.G. designed research; N.O., X.J.C., and K.D.I.S. performed research; N.O., X.J.C., K.D.I.S., and S.W.-G. analyzed data; N.O., X.J.C., S.W.-G., and J.D.E.G. wrote the paper.

This work was supported by NIH Grant R01-MH-080344 to J.D.E.G. We thank Christina Triantafyllou, Sheeba Arnold, and Steve Shannon at the Martinos Center for help with imaging protocols; Ayesha Hameed, Kai Qiu, Larissa Estrada, Tania Abedian, Sudhandra Sudanderam, and Natalie Rubinstein for help with data collection; and the participants and their families.

The authors declare no competing financial interests.

Correspondence should be addressed to Noa Ofen, Institute of Gerontology, 257 Knapp Building, 87 East Ferry Street, Detroit, MI 48202. E-mail: noa.ofen@wayne.edu.

DOI:10.1523/JNEUROSCI.1082-11.2012

Copyright $\odot 2012$ the authors $\quad 0270-6474 / 12 / 3210012-09 \$ 15.00 / 0$
}

jectories of specific brain regions and memory processes (Menon et al., 2005; Chiu et al., 2006; Ofen et al., 2007; Paz-Alonso et al., 2008; Chai et al., 2010; Ghetti et al., 2010; Maril et al., 2010). There is prolonged functional maturation of prefrontal and parietal cortices for successful encoding of vivid memories for scenes (recollection), but early maturation of those regions for encoding of less vivid memories for scenes (familiarity) (Ofen et al., 2007). The MTL appears to mature early in its contribution to both vivid and less vivid memories for simple scenes (Ofen et al., 2007), but mature more slowly in its support for vivid memories of complex scenes (Chai et al., 2010) and contextually rich memories (source memory) (Ghetti et al., 2010).

Only one study has examined the development of neural systems associated with successful retrieval of memories (PazAlonso et al., 2008). This study examined veridical and false memories for highly related words in children and adults and found age-related differences in MTL, prefrontal, and parietal activations. Successful memory retrieval with this sort of task involves a complex interaction between semantic knowledge of words, retrieval, and strategic monitoring (Roediger et al., 2001). Here we examined the development of successful memory retrieval for scenes, a domain of memory that is less complicated by parallel language and strategic development.

The objective of this study was to discover whether brain regions that are functionally associated with successful memory retrieval for scenes change across development from ages 8 to 21 . We hypothesized that, similar to successful memory encoding, activations in prefrontal and parietal regions associated with successful retrieval would increase with age. A hypothesis about 
MTL activations was less clear, because previous reports did not find age-related change in MTL activations for successful encoding of scenes (Ofen et al., 2007), but did find age-related increasing activations for retrieval of highly related words (Paz-Alonso et al., 2008). We also examined whether there are age-related changes in retrieval-related connectivity between the MTL and neocortical regions associated with the maturation of successful memory retrieval.

\section{Materials and Methods}

Participants. Eighty volunteers, ages 8-21 years old, were recruited from the local community and provided informed consent as required by a Massachusetts Institute of Technology Institutional Review Boardapproved protocol. Data from three participants (8-year-old male, 8 -year-old female, and 11-year-old male) were removed due to excessive movement that resulted in them not being able to contribute a complete data set in the scanning session. Data from other participants were excluded due to (1) behavioral noncompliance (i.e., performance was so poor that it appeared that participants were not performing or understanding the task; three participants, 14-year-old female, 17-year-old female, and 21-year-old male), (2) not completing the study (two 8-yearold males), and (c) technical difficulties when collecting behavioral responses in the scanner (three participants, 8-year-old female, 12-year-old female, and 14-year-old male). Neuroimaging data are thus presented for 69 participants ( 35 males; age range, $8-21$ years old; mean age, $14.7 \pm 4.2$ years) who met data quality criteria. All participants were right handed, had normal or corrected to normal visual acuity, and were screened for histories of psychiatric or developmental disorders. IQ estimates were obtained for each participant based on the Kaufman Brief Intelligence Test (second edition). The mean composite standard (normed to control for chronological age) IQ score across participants was 117.2 \pm 14.3 (SD). Importantly, participants' age and IQ did not correlate $(r=0.03$, $p=0.80$ ), indicating that in this sample of participants there was no confound of differences in IQ between younger and older participants. Participants 18 years or older were paid $\$ 20$ per hour for their participation. Participants younger than 18 years received a bookstore gift certificate with a value of $\$ 20$ per hour for their participation.

Materials. Two hundred and eighty pictures of indoor and outdoor scenes were used and divided into lists of 28 pictures (each comprised of 14 indoor and 14 outdoor scenes). Five lists were presented during the study, and the remaining five lists were presented as foils during the scanned recognition-memory test. List presentation order was counterbalanced across participants, such that pictures were presented equally often as study and test items across participants. Eight additional cartoon images of animals were used in a short practice period before the beginning of study.

Memory task. Participants studied 140 pictures of indoor and outdoor scenes. Each picture was presented for $2.5 \mathrm{~s}$. Participants were explicitly instructed to memorize the scenes for a later memory test. During the study phase, participants judged whether each picture depicted an indoor or outdoor scene, and indicated their judgment by pressing one button on the computer keyboard with their right index finger to indicate an indoor scene or another button with their left index finger to indicate an outdoor scene. The instructions (indoor/outdoor) appeared on the bottom of the screen, prompting participants to use the appropriate button press. Immediately following the study phase, participants were placed in an MRI scanner and given a recognition test consisting of 140 old and 140 new pictures presented on a screen. Each scene was presented on the screen for $3 \mathrm{~s}$, followed by a $1 \mathrm{~s}$ fixation cross. Variable intertrial intervals $(2-8 \mathrm{~s})$ were used to increase fMRI measurement reliability. For each picture, participants judged whether they had seen it before in the study phase (old) or not (new). Participants were instructed to take as much time as they needed to make their most accurate response, but to try to respond to each of the trials as soon as they had reached a decision. Intentional memory instructions were used to minimize the possibility that the older, compared to younger participants, would expect a later memory test and more voluntarily use intentional mnemonics strategies. Furthermore, the instructions emphasized accu- racy to assure that the younger participants attempt to reach an accurate retrieval decision. Studied items that elicited an old response were categorized as a HIT; studied items that elicited a new response were categorized as a MISS. New items (foils) that elicited a new response were categorized as a correct rejection (CR); new items that elicited an old response were categorized as a false alarm (FA). Participants were highly accurate (96.9\%) in making indoor/outdoor judgments during the study phase (on average, $135.6 \pm 4.2$ recorded correct responses from 140 trials). There was, however, a positive correlation between age and accuracy in the encoding task $(r=0.50, p<0.001)$, suggesting that younger participants were not properly attending to some of the items or pressing the wrong buttons for some items. To minimize potential age-related effects of attention during encoding from being carried over to the recognition test, only studied items that were correctly judged as indoor or outdoor were used in the imaging analysis. This minimized the influence of age on accuracy in the encoding task from influencing recognition test analyses. Reaction times for correct indoor/outdoor judgments during encoding $(1001 \pm 218 \mathrm{~ms})$ did not change with age $(r=0.10, p=0.43)$. Younger participants were more likely not to respond $(r=0.43, p<$ $0.001)$ or to press two different buttons for an item $(r=0.32, p=0.006)$ during the recognition test. The items for which either no response or more than one response occurred were omitted from the imaging analysis.

MRI data acquisition. Data were acquired on a 3T Tim Trio Siemens scanner using a 12-channel head coil. Before the functional scans, we acquired T1-weighted whole-brain anatomy images (MPRAGE sequence, $256 \times 256$ voxels, $1 \times 1.3 \mathrm{~mm}$ in-plane resolution, $1.3 \mathrm{~mm}$ slice thickness). Functional images were obtained in 32 -mm-thick slices (with $0.8 \mathrm{~mm}$ gap) oriented parallel to the line connecting the anterior and posterior commissures, covering the entire brain (echoplanar T2* weighted images; $3.1 \mathrm{~mm}$ in-plane resolution; repetition time, $2 \mathrm{~s}$; echo time, $30 \mathrm{~ms}$; flip angle, $90,64 \times 64$ voxels). To minimize effects of scanner signal stabilization, the first four images were omitted from all analysis. The memory task was imaged in five functional runs, each with 56 picture stimuli. Functional runs lasted $4 \mathrm{~min}$ and $56 \mathrm{~s}$, in which 144 images were collected. Foam pads were used to restrict head movement.

Imaging data analyses. SPM5 (Wellcome Department of Imaging Neuroscience, London, UK; http://www.fil.ion.ucl.ac.uk/spm/software/spm5) was used in all analyses. Images went through motion correction using sinc interpolation and normalized to the T2 Montreal Neurological Institute (MNI) template. Normalization to a common stereotactic space was used to allow comparison of activations across participants; such normalization has been shown to be appropriate for children age 7 and older (Burgund et al., 2002). Finally, images were spatially smoothed with an isotropic Gaussian kernel of $6 \mathrm{~mm}$ full-width at half-maximum.

All functional data were subjected to artifact detection using custom software (http://web.mit.edu/swg/software.htm). From each participant, an image was defined as an outlier if movement from a preceding image exceeded $0.5 \mathrm{~mm}$ in any of the three translation directions and/or 0.01 radians of rotation. An image was also defined as an outlier if the global mean intensity in the image was $>3$ SDs from the mean image intensity for the run in which it was collected. Overall, $2.3 \pm 3.0 \%$ (mean $\pm \mathrm{SD}$ ) of the images were defined as outliers (with a maximum $16.1 \%$ in one participant). A single regressor for each outlier image was included in the first level model (described below) to control for outlier images effects on estimated activations. Analysis of the movement parameters obtained during realignment (three translation and three rotation, mean of the means of each of five runs) showed that movement increased with age, as evident in a negative correlation between age and two movement dimensions $(x$-axis translation, $r=-0.27, p=0.03 ; y$-axis translation, $r=$ $-0.32, p=0.01$; movement in the $z$-axis translation and in the three rotational dimensions did not change reliably with age, $0.03<|r|<0.13$, $p$ values $>0.29$ ). Movement parameters were not correlated with stimulus presentation across participants (for any of the six movement parameters with either HIT or CR; mean, $|r|$ values $<0.03$; max, $|r|$ values of $0.17)$. Therefore, to minimize artifacts due to differences in movement across ages, movement parameters (the six movement parameters assessed during realignment) were included as nuisance covariates in participants' statistical models (first-level analysis). Inclusion of motion 
parameters ensured that the variance related to residual head motion was explicitly modeled, and hence would not result in false positives.

Individual general linear model-based analyses were conducted in MNI space. Models included regressors of interest generated by convolving task events (HIT, CR) with a canonical model of the homodynamic response function (HRF) as implemented in SPM5, including temporal derivative. A canonical model of the HRF were used, as previous studies found minimal differences in HRF time courses between children and adults (Kang et al., 2003). Additional regressors and their temporal derivatives modeled other task events (MISS, FA) and invalid trials (incorrect response during encoding, more than one or no response during encoding). Additional regressors modeled motion (as described above) and outlier images (one regressor per outlier image). Individual subject's effects were estimated using a fixed-effects model across the five runs. Linear combination of the HIT minus CR regressors was used in defining contrasts of interest for successful retrieval. Memory-related activations were also assessed using a linear combination of HIT minus MISS, FA minus CR, and CR minus FA contrasts. Group analyses that included FA were restricted to participants having $>17$ FA trials $(n=56 ; 30$ males; age range, $8-21$ years old; mean age, $15.0 \pm 4.1$ years). Contrasts constructed at the single participant level were then input into a second-level group analysis using a random-effects model. Reported clusters survived thresholds of an uncorrected $p<0.001$ at the voxel level and a familywise error (FWE) correction (as implemented in SPM, using Gaussian Field Theory) of $p<0.05$ at the cluster level. A one-sample $t$ test was performed on the contrast HIT $>$ CR. Next, age effects for successful retrieval (HIT > CR) were investigated across the whole brain regressing age as a continuous variable. In addition, age effects were also examined at a more lenient threshold of $p<0.001$ and a cluster extent of 50 contiguous voxels. In a complementary analysis, we used functionally defined ROIs derived from the group-level HIT $>$ CR activations map to examine, across participants, the correlations of successful retrieval activations (HIT $>$ CR) with age and recognition memory accuracy. In defining ROIs from the group-level map of HIT $>$ CR, we used a corrected voxel-level FEW threshold of 0.001 , which allowed for identifying limited extent clusters as ROIs.

Because of the known importance of MTL regions for memory, and known problems of susceptibility in the MTL region, we tested the a priori hypotheses that these regions are involved in memory retrieval. ROIs within the MTL were defined by small volume correction on the group HIT > CR activation map using an anatomically defined MTL mask comprising the left and right hippocampi and left and right parahippocampal gyri (using the Wake Forest University Pickatlas tool). Reported clusters survived an uncorrected $p<0.001$ at the voxel-level and FWE correction of $p<0.05$ at the cluster-level. In ROI analyses, data were extracted from individual participant's contrast images and subjected to a test of correlation with age as a continuous variable.

To examine possible developmental changes in functional connectivity of MTL during successful memory retrieval, we conducted a psychophysiological interaction (PPI) analysis (Friston et al., 1997). We identified MTL seed ROIs for the PPI analysis based on MTL regions that were active in the group HIT $>$ CR map (described above). Seed ROIs were created as $4 \mathrm{~mm}$ spheres centered on peak activations in left parahippocampal gyrus (PHG; $-18,-30,-14)$, right PHG $(18,-34,-10)$, and left anterior MTL $(-16,2,-16)$. The PPI analysis compared the temporal correlation (or functional "coupling") between the MTL seed regions and other brain regions (physiological component) during different memory conditions (psychological component). The PPI design matrix contained three columns of variables: (1) a "psychological" variable that represents the contrast of two conditions (Hit > CR), (2) a time-series "physiological " variable that represents the time course of the source (seed) region, and (3) an interaction variable that represents the interaction of the psychological and physiological, respectively, variables (1) and (2). The regression coefficient for the interaction term provides a measure of PPI; a correlation (or covariance) in activation between the MTL seed region and the identified "coupled" region that is significantly different between memory conditions ( HIT $>C R$ ) yields a significant PPI effect. Individual $\beta$ images of the interaction term were entered into a second-level whole-brain correlation analysis with age as a regressor
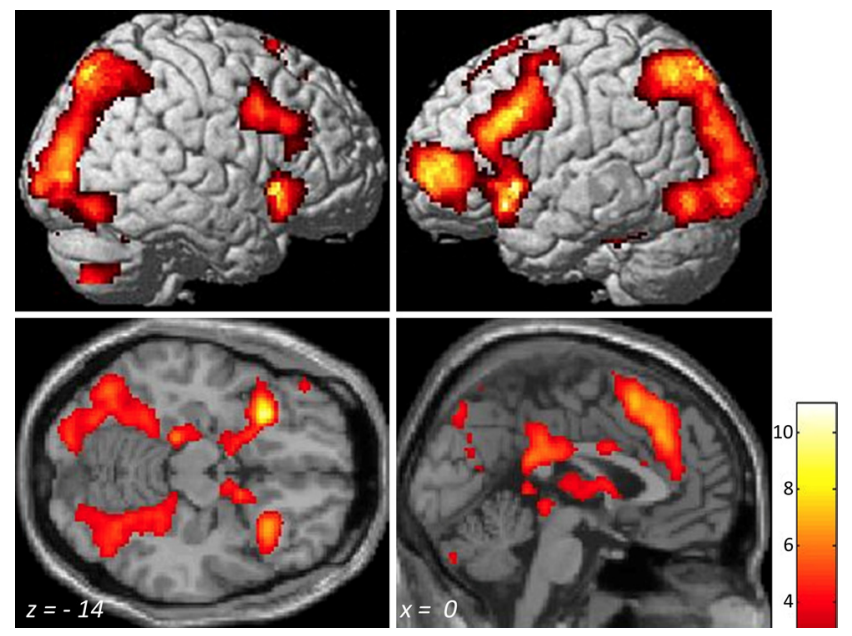

Figure 1. Successful retrieval activation (HIT $>$ CR) across all 69 participants (ages $8-21$ years). Activation maps are rendered on images from a standard brain right and left lateral views (top) and on horizontal and sagittal sections (bottom). MNI coordinates are presented at the bottom of each section. Voxel-level threshold, $p<0.001$; corrected at the cluster level with few, $p<0.05$. A $t$ value scale is presented at the bottom right.

Table 1. Successful retrieval effects

\begin{tabular}{|c|c|c|c|c|c|c|}
\hline & \multirow[b]{2}{*}{ BA } & \multicolumn{3}{|c|}{ MNI coordinates } & \multirow{2}{*}{$\begin{array}{l}\text { Peakt } \\
\text { value }\end{array}$} & \multirow{2}{*}{$\begin{array}{l}\text { No. of } \\
\text { voxels }\end{array}$} \\
\hline & & $x$ & $y$ & $z$ & & \\
\hline \multicolumn{7}{|l|}{ Across all participants } \\
\hline Left caudate & n.a. & -10 & 12 & 0 & 11 & 8983 \\
\hline Left inferior frontal gyrus & 47 & -30 & 20 & -8 & 10.71 & \\
\hline Right caudate & n.a. & 8 & 12 & 2 & 9.76 & \\
\hline Right posterior cingulate/preuneus & 30 & 20 & -58 & 24 & 9.75 & 18929 \\
\hline Left posterior cingulate/preuneus & 30 & -14 & -60 & 16 & 9.03 & \\
\hline Right precuneus & n.a. & 16 & -68 & 44 & 8.82 & \\
\hline Right inferior frontal gyrus/insula & $47 / 11$ & 32 & 24 & 0 & 8.92 & 1044 \\
\hline Left/right medial/superior frontal gyrus & $9 / 8$ & -4 & 34 & 34 & 8.4 & 2920 \\
\hline Right cerebellum & n.a. & 6 & -82 & -30 & 6.79 & 581 \\
\hline Left cerebellum & n.a. & -10 & -72 & -30 & 6.14 & \\
\hline Right inferior frontal gyrus & 9 & 42 & 6 & 32 & 6.29 & 927 \\
\hline Right middle frontal gyrus & 46 & 48 & 22 & 30 & 5.25 & \\
\hline Right/left cingulate gyrus & 24 & 6 & 4 & 30 & 5.47 & 179 \\
\hline Right cerebellum & n.a. & 36 & -68 & -50 & 5.39 & 244 \\
\hline \multicolumn{7}{|l|}{ Within the MTL } \\
\hline Left parahippocampal gyrus & $35 / 28 / 19$ & -18 & -30 & -14 & 6.09 & 184 \\
\hline Right parahippocampal gyrus & $35 / 36 / 37$ & 18 & -34 & -10 & 4.98 & 117 \\
\hline Left entorhinal cortex/amygdala & $34 / 28$ & -16 & 2 & -16 & 4.24 & 58 \\
\hline \multicolumn{7}{|l|}{ Increased with age } \\
\hline Left middle/superior frontal gyrus & 6 & -32 & 10 & 64 & 4.43 & $54^{a}$ \\
\hline Left inferior frontal gyrus & 47 & -46 & 24 & -10 & 4.38 & $95^{a}$ \\
\hline Right inferior frontal gyrus & 47 & 56 & 22 & 0 & 4.14 & $53^{a}$ \\
\hline Left superior parietal lobule & $7 / 40$ & -24 & -70 & 54 & 4.06 & 179 \\
\hline Left/right caudate & n.a. & -2 & -6 & -4 & 3.95 & $60^{a}$ \\
\hline Right inferior frontal gyrus & 47 & 34 & 24 & -18 & 3.59 & $52^{a}$ \\
\hline
\end{tabular}

${ }^{a}$ Increases with age did not survive FWE cluster-level correction. n.a., Not applicable.

variable to identify developmental changes in MTL PPI effects. Wholebrain PPI analyses of the three MTL seeds were constrained using a binary mask from the whole-brain analysis of age-related changes in successful retrieval (HIT $>$ CR). This procedure allowed us to test for developmental influences on PPI effects between MTL seeds and neocortical regions that showed developmental effects in successful retrieval activations. Small volume correction was performed using this mask (FWE cluster-level threshold of $p<0.05$ ). We also examined age-related changes in PPI effects of the MTL seeds at the whole-brain level ( $p<0.001$, voxel-level uncorrected; cluster extent, $>50$ contiguous voxels). 


\section{Results}

\section{Behavior}

Across all participants, $53 \pm 13 \%$ (mean \pm SD) of the studied scenes were recognized as old (HIT rate), and $24 \pm 12 \%$ of the new scenes presented at the test (foils) were falsely classified as old (FA rate). HIT rate increased significantly with age $(r=0.43, p<$ $0.001)$, whereas CR rate did not change reliably with age $(r=-0.18$, $p=0.14$ ) (see Fig. 2a). The difference between the age-related correlations for HIT and CR was significant $(z=5.7, p<0.001$, twotailed). Participants' response times for HIT (1612.1 $\pm 226.6 \mathrm{~ms}$ ), MISS $(1684.7 \pm 287.1 \mathrm{~ms}), \mathrm{CR}(1630.7 \pm 240.0 \mathrm{~ms})$, and FA (1794.2 $\pm 319.9 \mathrm{~ms})$ did not change significantly with age $(|r|$ values $<0.19$, $p$ values $>0.12$ ).

\section{Imaging}

Successful retrieval across all participants

Across all participants, successful retrieval (contrast HIT $>$ CR) was associated with significant activations in prefrontal, parietal, and temporal regions, as well as in subcortical regions (Fig. 1; Table 1). Across all participants, three regions within the MTL showed successful retrieval activations (Table 1).
Age-related changes in successful retrieval

A significant age-related increase in activation for successful retrieval was found in left parietal cortex with peak coordinates in the superior parietal lobule (SPL; $p<0.001$, voxel level; clustercorrected FWE, $p<0.05$ ) (Table 1; Fig. $2 c$ ). The majority of voxels in this parietal cluster were within the superior parietal lobule $(72 \%$ in $7 \mathrm{~A}, 13 \%$ in $7 \mathrm{P}$; a small portion were within the superior part of the inferior parietal cortex, PGa, 12\%; PFm, $0.6 \%$ ) as identified using probabilistic cytoarchitectonic maps from the SPM Anatomy Toolbox (Eickhoff et al., 2005). In addition, at a more liberal threshold, activations associated with successful retrieval increased with age in bilateral prefrontal neocortices and the left caudate $(p<0.001$, voxel level; cluster extent, $>50$ contiguous voxels) (Fig. $2 b, c$; Table 1). HRF time courses from the left superior parietal lobule demonstrate increased differentiation between HIT and CR from children (ages 8-12) to adolescents (ages 13-17) to adults (ages 18-21) (Fig. 2d).

In a complementary investigation of age effects, we used functionally defined ROIs form the group HIT $>$ CR activation map. In a left parietal ROI (peak coordinates, $-14,-60,16$ ) activa-
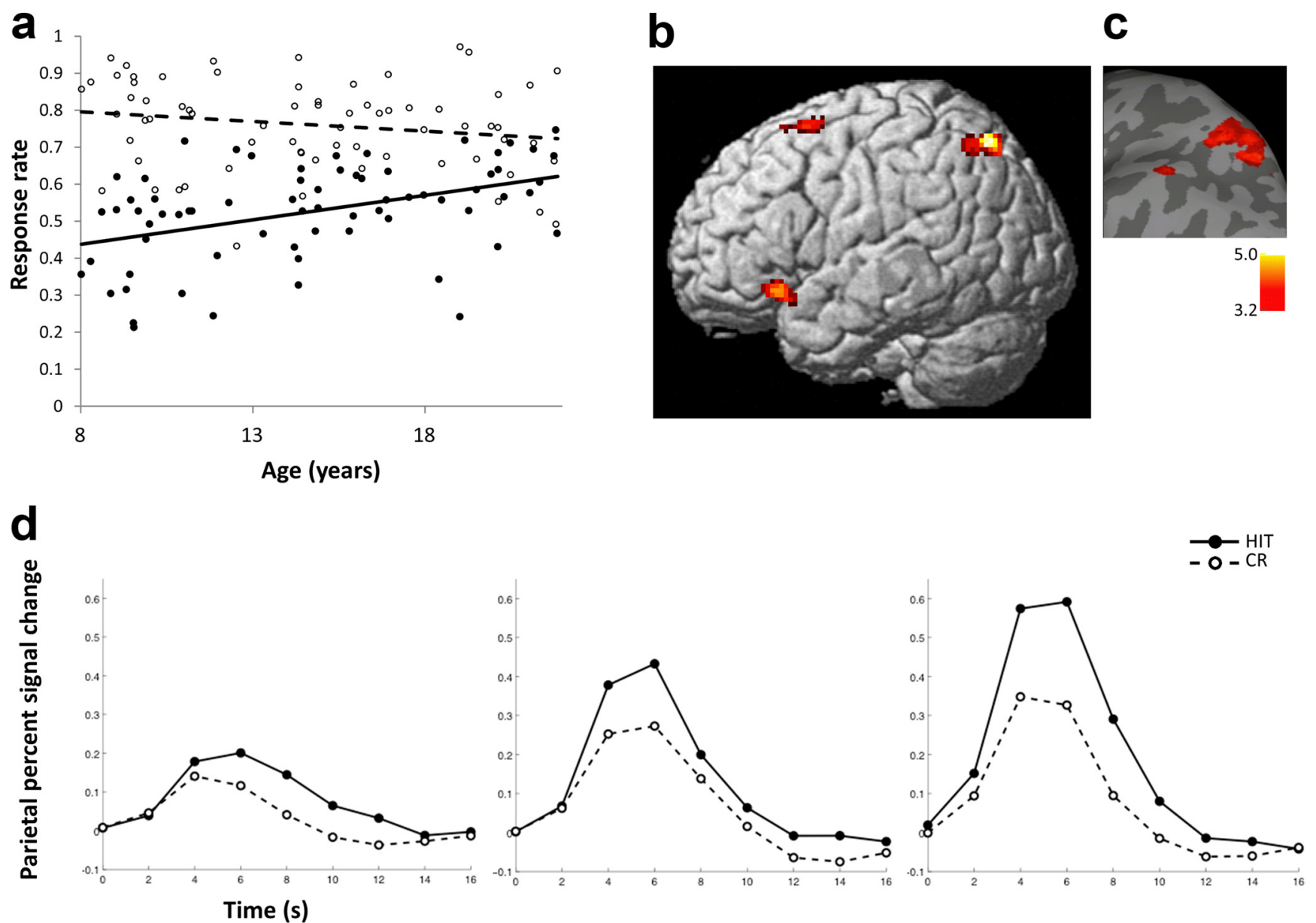

Figure 2. Age-related changes in recognition memory and brain activations associated with successful retrieval. $\boldsymbol{a}$, HIT rate improved significantly with age (individual values in closed circles; regression with age, solid line), and (R accuracy did not change significantly with age (individual values in open circles; regression with age, dashed line). $\boldsymbol{b}$, Activations associated with retrieval success (rendered on a standard brain's left lateral view) increased with age in left parietal and frontal regions as indicated by regression analysis using age as a continuous variable across participants. Voxel-level threshold, $p<0.001$; cluster extent, $>50$ contiguous voxels. $\boldsymbol{c}$, For visual presentation, parietal activations depicted in $\boldsymbol{b}$ were overlaid on a surface based representation of the MNI canonical brain using the SPM SurfRend Toolbox (http://spmsurfrend.sourceforge.net). A t value scale is presented at bottom right. $\boldsymbol{d}$, Time courses for the HIT and CR conditions in left superior parietal cortex were extracted for each participant and averaged across participants in three age groups corresponding to children (left; $n=27 ; 8-12$ years old), adolescents (middle; $n=$ 24; 13-17 years old), and adults (right; $n=18 ; 18-21$ years old). HIT, Closed circles, solid line; (R, open circles, dashed line. 
tions significantly increased with age $(r=0.25, p=0.04)$. Similarly, activation increased with age in the right caudate ROIs (peak coordinates, $8,12,2 ; r=0.30, p=0.01$ ), and marginally in the left caudate (peak coordinates, $-10,12,0 ; r=0.23, p=0.06$ ). In one ROI in the medial frontal gyrus, activation increased with age (peak coordinates, $-4,34,34 ; r=0.24, p=0.04$ ). In other left and right frontal ROIs (peak coordinates, left, $-30,20,-8 ;-42$, 18,$30 ;-42,46,2$; right, 32, 24, 0 ) activations did not change with age $(|r|$ values $<0.18$, $p$ values $>0.16)$. Recognition memory accuracy (average HIT rate and CR rate) increased with age $(r=$ $0.30, p=0.01$ ), but was not a significant predictor of activation in any of the ROIs defined by group activations $(|r|$ values $<0.18, p$ values $>0.14)$. Age-related increases in HIT $>C R$ activation within ROIs remained significant (right caudate, $r=0.27, p=$ 0.03 ) or approached significance (left parietal, left caudate, medial frontal gyrus; $|r|$ values $>0.20$, $p$ values $<0.10$ ) when controlling for individual differences in recognition memory accuracy. Thus, both whole-brain analysis and ROI methods of assessing age effects in successful retrieval activations revealed developmental effects in left superior parietal cortex and caudate, and whole-brain analysis at a more lenient threshold suggested developmental effects in bilateral prefrontal cortices.

In contrast to the age-related changes described above, there were no age-related correlations with successful retrieval activations in MTL regions $(|r|$ values $<0.17$, $p$ values $>0.15)$ (Fig. 3 ). In addition, there were no age-related decreases in activations associated with successful retrieval.

\section{Retrieval-related functional connectivity between MTL and prefrontal cortex increased with age}

First, we identified age-related increases of differential functional connectivity assessed with PPI for successful retrieval (HIT vs CR) between the three MTL seeds and the regions in which activations for successful retrieval increased with age (MTL PPI effects restricted to regions of HIT $>$ CR activations increased with age). Across all participants, there was significant age-related change in PPI between the left PHG and a region in the left inferior frontal gyrus (IFG; $r=0.46, p<$ 0.001) (Fig. 4; Table 2). Thus, the PPI effects demonstrated reliable change in the pattern of MTL-IFG connectivity across the HIT and CR trials as a function of age. Furthermore, we tested age-related changes in left PHG-left IFG connectivity separately for HIT and CR trials. Left PHG-left IFG connectivity during CR responses significantly decreased with age $(r=-0.38, p=0.001)$, whereas left PHGleft IFG connectivity during HIT trials did not change with age $(r=0.10, p=0.42)$. PPI between the right PHG and left IFG also changed with age $(r=0.40, p<$ $0.001)$. No significant developmental effects were found in PPI of the left anterior MTL. Second, we examined agerelated changes in the PPI of the three MTL seeds at the whole-brain level (not restricted to regions of HIT $>$ CR activations increased with age; $p<0.001$, voxel level; cluster extent, $>50$ contiguous voxels). There was a significant agerelated change in PPI between the left PHG and the same region in the left in- ferior frontal gyrus that was identified when we tested for PPI effects with a mask (described above in this section) (Table 2). No brain regions showed age-related changes in PPI with the right PHG, or with the left anterior MTL using this threshold for the whole-brain-level analysis.

\section{Additional retrieval contrasts}

Across all participants, there were significant activations for the contrast HIT > MISS (recognition contrast) in prefrontal and parietal cortical regions and in MTL and subcortical regions (Table 3). Activations for HIT > MISS increased with age in the left inferior parietal lobule/supramarginal gyrus, left inferior frontal gyrus, superior parietal cortex, and cerebellum $(p<0.001$, voxel level; cluster extent, $>50$ contiguous voxels) (Table 3 ). There was overlap in the left superior parietal and left inferior frontal clusters that showed age-related increase for HIT $>$ CR and HIT $>$ MISS (Table 3; Fig. 5). Furthermore, similar to what was found for HIT $>\mathrm{CR}$, there were no age-related changes in HIT $>$ MISS activations within the MTL $(|r|$ values $<0.06$, $p$ values $>0.60)$.

Across all participants, there were significant activations for the contrast FA $>$ CR (false memory of new scenes) in prefrontal and parietal cortical regions and in subcortical regions (Table 4). Activations for FA $>$ CR increased with age in the left and right inferior frontal gyri and in the left posterior cingulate gyrus, left and right superior parietal cortices, cerebellum, caudate, and left insula ( $p<0.001$, voxel level; cluster extent, $>50$ contiguous voxels) (Table 4 ). There was overlap in the left superior parietal and left inferior frontal clusters that showed age-related increases for HIT $>$ CR and FA $>$ CR (Table 4; Fig. 5). Age-related increases in activations for $\mathrm{FA}>\mathrm{CR}$ were found in similar regions that showed age-related increases for HIT $>$ CR. Although in this study rates of CR and FA did not change with age, age-related increases in false memory are commonly found when richly associative verbal memory is tested (Paz-Alonso et al., 2008). The overlap of age-related increases for HIT $>$ CR and for FA $>$ CR is in line with an interpretation that age-related increases in recognition memory accuracy coincide with age-related increases in

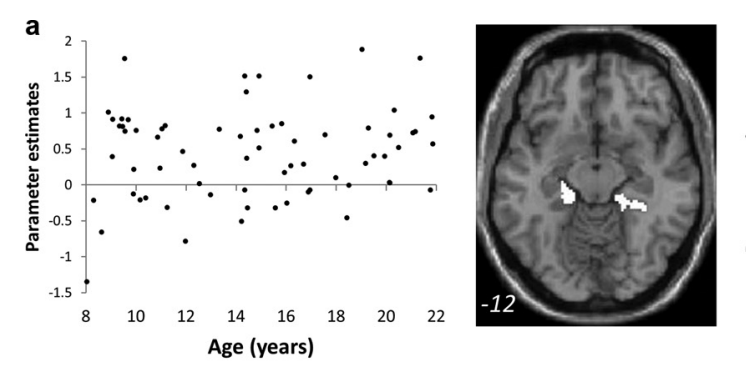

b

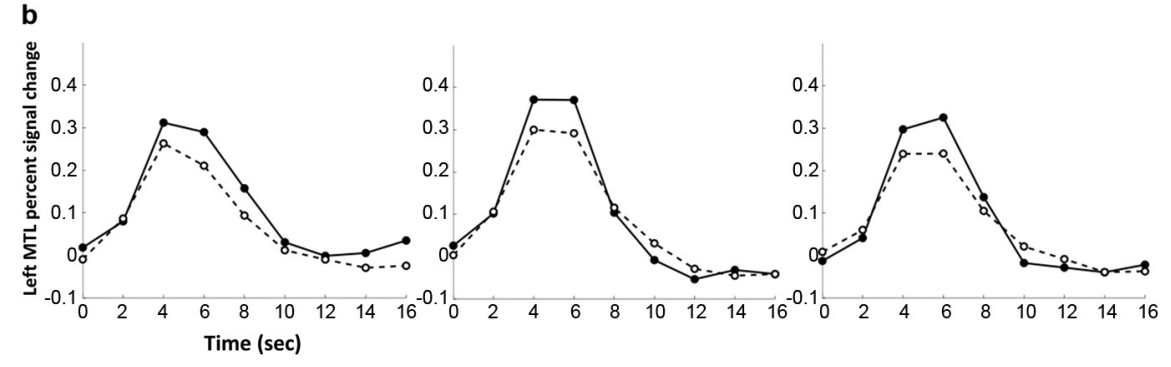

Figure 3. Activations associated with successful retrieval did not change with age in MTL. $\boldsymbol{a}$, Left, Right, Plots of correlations of parameter estimates values extracted from individual participants in the contrast HIT $>$ CRfrom left and right MTL ROIs. Middle, The binary masks of left and right ROIs within the MTL are depicted on an axial brain image. The MNI $z$-coordinate is presented in the bottom of the image. $\boldsymbol{b}$, Time courses of retrieval success activations in. Time courses for the HIT and CR conditions in the left MTL in children, adolescents, and adults are shown. Description of age group and symbols are as described in Figure $2 d$. 

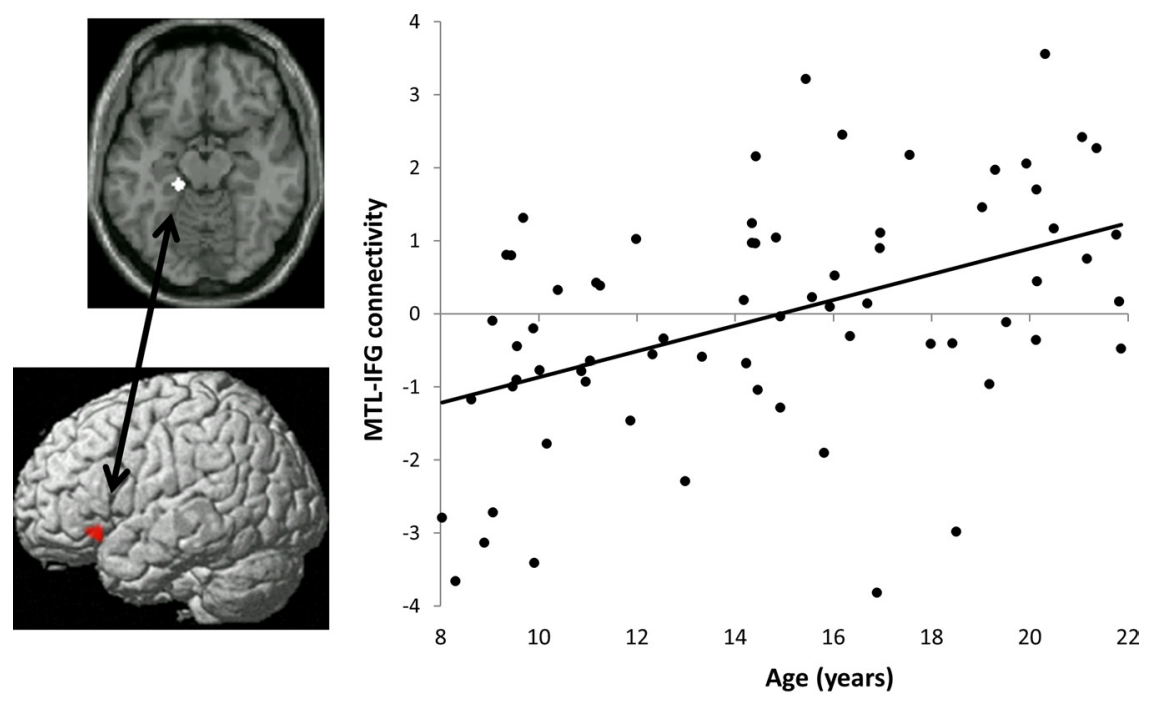

Figure 4. PPI for successful retrieval (difference between HIT and (R responses) between MTL and prefrontal cortex changed with age. Top left, PHG seed used in the PPI analysis (overlaid on an axial standard brain's image). Bottom left, Left IFG cluster that exhibited age-related changes in differential functional connectivity for HIT versus CR (PPI) with the PHG seed (rendered on a standard brain's left lateral view). Right, Parameter estimate of the PPI between left PHG and left IFG, plotted against age. Similar age-related changes in PPI were observed between right PHG and left IFG.

Table 2. MTL connectivity changed with age

\begin{tabular}{|c|c|c|c|c|c|c|}
\hline & \multirow[b]{2}{*}{ BA } & \multicolumn{3}{|c|}{ MNI coordinates } & \multirow{2}{*}{$\begin{array}{l}\text { Peak } t \\
\text { value }\end{array}$} & \multirow{2}{*}{$\begin{array}{l}\text { No. of } \\
\text { voxels }\end{array}$} \\
\hline & & $x$ & $y$ & $z$ & & \\
\hline \multicolumn{7}{|l|}{$\begin{array}{l}\text { Within regions showing maturation of } \\
\text { successful retrieval effects }\end{array}$} \\
\hline \multicolumn{7}{|l|}{ MTL seed region } \\
\hline \multicolumn{7}{|l|}{ Left PHG } \\
\hline \multicolumn{7}{|l|}{ Right PHG } \\
\hline Left inferior frontal gyrus & 47 & -44 & 24 & -6 & 3.99 & 7 \\
\hline \multicolumn{6}{|l|}{ Left anterior MTL } & \\
\hline \multicolumn{7}{|l|}{ Whole brain analysis } \\
\hline \multicolumn{7}{|l|}{ MTL seed region } \\
\hline \multicolumn{7}{|l|}{ Left PHG } \\
\hline Left inferior frontal gyrus & 47 & -44 & 24 & -10 & 4.39 & $83^{a}$ \\
\hline $\begin{array}{l}\text { Left/right, medial frontal/ } \\
\text { precentral }\end{array}$ & 6 & 12 & -28 & 64 & 4.39 & $90^{a}$ \\
\hline \multicolumn{7}{|l|}{ Right PHG } \\
\hline \multicolumn{7}{|l|}{ None } \\
\hline \multicolumn{7}{|l|}{ Left anterior MTL } \\
\hline
\end{tabular}

MTL seed region coordinates are listed in Table 1.

${ }^{a}$ Increases with age did not survive FWE cluster-level correction.

false memory. Across all participants there were significant activations for the contrast CR $>$ FA (accurate novelty monitoring) in the anterior MTL and in parietal and medial frontal regions that are typically associated with the default network (Table 4). There were no changes with age for contrast CR $>$ FA.

The main focus of this study was to identify developmental effects in activations for retrieval success (contrast HIT $>\mathrm{CR}$ ). Because the other contrasts with FA and MISS trials involved the HIT and CR trials, it might be expected that similar regions of activation would be revealed as in the HIT $>$ CR contrast. These analyses, however, support the view that similar developmental factors support both successful objective memory retrieval (HIT > CR) and also subjective memory retrieval (Wheeler and Buckner, 2003) of "oldness" (HIT > MISS) or "newness" (FA $>$ CR).

\section{Discussion}

This study revealed the development of declarative memory systems associated with successful retrieval from memory for scenes in children, adolescents, and young adults. Older participants were more accurate in recognition memory decisions, and specifically in recognizing previously presented scenes. Successful retrieval across all ages was associated with activations in frontal, parietal, and MTL brain regions that are consistently activated for successful retrieval in adults (Konishi et al., 2000; Montaldi et al., 2006; Spaniol et al., 2009). Successful retrieval activations increased with age in left parietal, bilateral caudate, and bilateral frontal regions. In contrast, successful retrieval activations in the MTL did not change with age, although retrieval-related PPI connectivity between left and right parahippocampal gyri and the left IFG changed with age.

Although the influence of age on brain activation was examined only for scenes that were accurately encoded and accurately retrieved, it is likely that retrieved memories for studied scenes were less vivid or detailed in younger participants. To minimize demands on the youngest participants, we did not collect a second measure of memory strength for each response to distinguish between more vivid, or recollected, memories versus less vivid, or familiar, memories. In a parallel study of encoding for similar scenes, there was no developmental influence for subsequent familiarity-based retrieval for scenes, but there were significant developmental influences both on accuracy for recollection-based retrieval for scenes, and also on activations in left parietal and bilateral prefrontal regions that were associated with subsequent recollection-based retrieval for scenes (Ofen et al., 2007). Therefore, the developmental differences found in parietal and frontal regions in the present study of retrieval are likely to reflect reduced details or information in the retrieved memories of younger participants.

Activation for successful retrieval grew across ages in bilateral ventral prefrontal cortex and left dorsal prefrontal cortex. The ventral prefrontal cortex is implicated in selection and retrieval of information (Thompson-Schill et al., 1997; Wagner et al., 2001; Thompson-Schill, 2003) and the maintenance of retrieved representations (Petrides et al., 1993; Rugg et al., 1999; Dobbins et al., 2002; Dobbins and Han, 2006). Age-related increases in successful retrieval activations in these regions may reflect older participants relying more on semantic knowledge or using better maintenance processes for successful retrieval than younger participants. Dorsal prefrontal cortex is also implicated in memory retrieval in adults (Spaniol et al., 2009) and may specifically contribute to retrieval of pictorial stimuli, rich in spatial information (Passingham and Sakai, 2004). Activations also increased with age in the left caudate, a region that is strongly connected with the left ventral prefrontal cortex (Barnes et al., 2010).

The strongest influence of age occurred in left parietal cortex. Activation for successful retrieval grew across ages primarily in the left SPL (BA7). This age-related growth of parietal activation for retrieval of memory for scenes is similar to developmental findings of memory for verbal information (Paz-Alonso et al., 2008). Multi- 
ple studies in adults report successful retrieval activations across left parietal regions in BA7, BA39, and BA40 (Cabeza et al., 2008). Most of these studies involved verbal stimuli, but activation for scenes increased during retrieval as a function of self-reported memory strength in left parietal cortex somewhat ventral to the region showing an age-related change in the present study (Montaldi et al., 2006). Increasing activation exhibited by adults in the present study may have reflected the increasing strength of memories retrieved from childhood to adulthood.

The role of parietal cortex in memory retrieval has been the focus of intense investigation (Cabeza, 2008; Ciaramelli et al., 2008; Vilberg and Rugg, 2008; Wagner et al., 2005) motivated by its ubiquitous activation in neuroimaging studies and by the lack of neuropsychological evidence for a critical role of parietal cortex in memory (Haramati et al., 2008). Studies in adults have suggested that left superior regions of the lateral parietal regions reflect top-down attentional processes for memory retrieval (Cabeza, 2008; Ciaramelli et al., 2008, 2010; Vilberg and Rugg, 2008). The extent to which these superior parietal activations reflect primary memory retrieval processes is unclear, because these regions are sensitive to expectations and decision biases more generally (O'Connor et al., 2010), but such attentional and retrieval effects may be separable (Hutchinson et al., 2009; Sestieri et al., 2010). It has been proposed that activations in superior parietal cortex (BA7) are associated with familiaritybased retrieval, whereas activations in inferior parietal cortex (BA39 and BA40) are associated with recollection-based retrieval (Yonelinas et al., 2005; Cabeza et al., 2008; Ciaramelli et al., 2008; Vilberg and Rugg, 2008). Given prior evidence that developmental influences are greater on recollection than familiarity at retrieval, it might have been expected that developmental differences would have occurred in more inferior parietal cortex instead of the more superior location, where the developmental influence was found. Developmental studies that more precisely control for recollection and familiarity may clarify this issue.

There were no developmental changes in MTL activations associated with successful retrieval, despite the finding that such activations were found across ages. Developmental changes in MTL activations associated with memory are inconsistent across studies. Age-related reductions in MTL activations were found during the encoding of scenes (Menon et al., 2005) and of certain verbal memories (Maril et al., 2010). Another study found no developmental changes in MTL activation for encoding that yielded recognition familiarity for simple and complex scenes and recollection for simple scenes, but age-related increases in activations in posterior parahippocampal gyrus that supported subsequent recollection of complex scenes (Chai et al., 2010). Similarly, age-related increases of MTL activations have been reported for formation (Ghetti et al., 2010) and retrieval of verbal memories, and with recognition of sentences in older but not in younger children (Chiu et al., 2006). Together, these findings suggest protracted maturation in some, but not all, MTL functions related to memory, but it is not yet clear what principles determine when MTL activations do or do not increase with age. In general, it appears that memory processes that involve detailed memories are associated with agerelated increases in MTL activation. The present findings are also consistent with a proposal of dissociable developmental trajecto-

Table 3. HIT > MISS (recognition effects)

\begin{tabular}{|c|c|c|c|c|c|c|}
\hline & \multirow[b]{2}{*}{ BA } & \multicolumn{3}{|c|}{ MNI coordinates } & \multirow{2}{*}{$\begin{array}{l}\text { Peak } t \\
\text { value }\end{array}$} & \multirow{2}{*}{$\begin{array}{l}\text { No. of } \\
\text { voxels }\end{array}$} \\
\hline & & $x$ & $y$ & $z$ & & \\
\hline \multicolumn{7}{|l|}{ Across all participants } \\
\hline Left inferior frontal gyrus/insula & $47 / 13$ & -28 & 20 & -8 & 8.84 & 7854 \\
\hline Right caudate & n.a. & 8 & 12 & 2 & 8.38 & \\
\hline Left caudate & n.a. & -10 & 10 & 0 & 8.03 & \\
\hline Left occipital lobe/parietal lobe & $18 / 19$ & -36 & -86 & -6 & 7.93 & 19779 \\
\hline Right precuneus & 7 & 26 & -68 & 30 & 7.59 & \\
\hline Right superior parietal lobe & 7 & 28 & -66 & 46 & 7.47 & \\
\hline Left medial frontal/anterior cingulate & $9 / 8 / 32$ & -4 & 38 & 32 & 7.44 & 1968 \\
\hline Right inferior frontal gyrus/insula & $47 / 13$ & 38 & 22 & -6 & 7.3 & 917 \\
\hline Left cerebellum & n.a. & -10 & -74 & -30 & 5.85 & 211 \\
\hline Right inferior/middle frontal gyrus & $46 / 45 / 10$ & 56 & 34 & 14 & 5.66 & 1211 \\
\hline Right inferior frontal gyrus & 9 & 42 & 6 & 32 & 5.02 & \\
\hline Right cerebellum & & 30 & -72 & -50 & 4.56 & 222 \\
\hline \multicolumn{7}{|l|}{ Within the MTL } \\
\hline Left entorhinal cortex/amygdala & $34 / 28$ & -16 & 2 & -16 & 5.52 & 315 \\
\hline Left hippocampus & n.a & -32 & -8 & -16 & 4.75 & \\
\hline Right parahippocampal gyrus & $36 / 37$ & 36 & -36 & -16 & 4.55 & 44 \\
\hline Right entorhinal cortex/amygdala & 34 & 14 & -6 & -16 & 4.33 & 60 \\
\hline \multicolumn{7}{|l|}{ Increased with age } \\
\hline $\begin{array}{l}\text { Left supramarginal gyrus/inferior } \\
\text { parietal lobe }\end{array}$ & 40 & -56 & -50 & 36 & 5.01 & 168 \\
\hline Left cerebellum & n.a. & -46 & -74 & -26 & 4.64 & $71^{a}$ \\
\hline Left inferior frontal gyrus & 47 & -48 & 30 & -8 & 4.34 & 171 \\
\hline Left superior parietal lobe & 7 & -36 & -64 & 56 & 4.29 & 140 \\
\hline
\end{tabular}

Regions in bold are also associated with age-related increases in the contrast HIT > CR. n.a., Not applicable. ${ }^{a}$ Increases with age did not survive FWE cluster-level correction. a $\mathrm{HIT}>\mathrm{CR}$

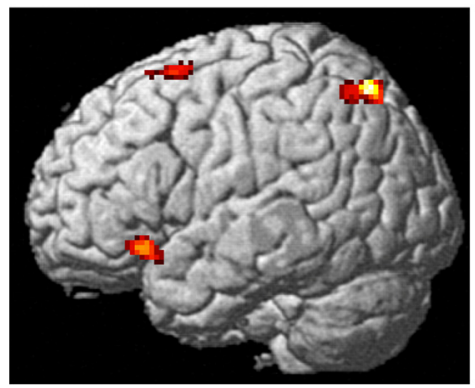

C FA $>$ CR

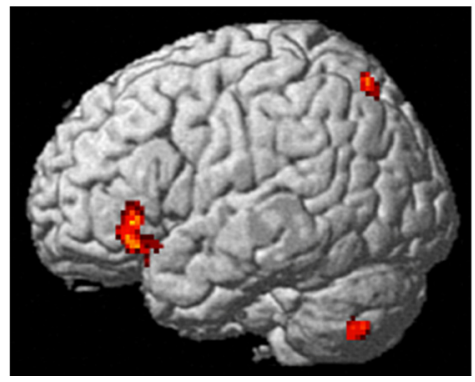

b $\quad$ HIT $>$ MISS
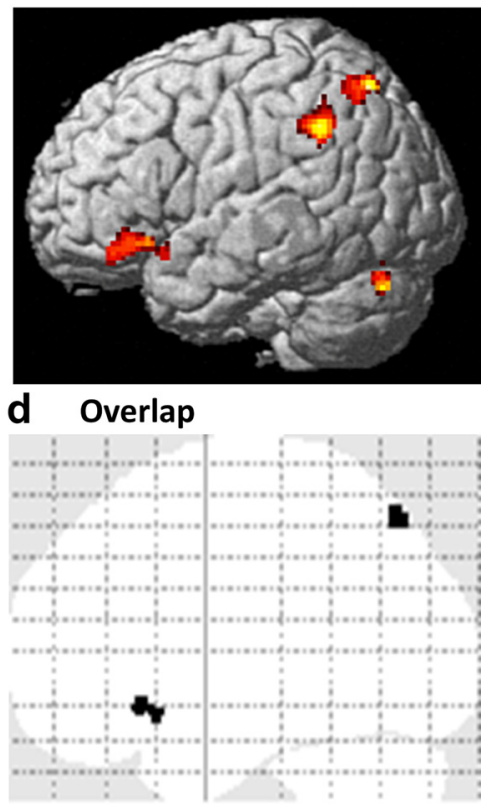

Figure 5. Similar age-related changes in retrieval contrasts HIT $>C R$, HIT $>$ MISS, and FA $>C R$. $\boldsymbol{a}-\boldsymbol{c}$, Activations associated with contrast HIT $>$ CR (successful retrieval; $\boldsymbol{a}$ ), contrast HIT $>$ MISS (accurate recognition; $\boldsymbol{b}$ ), and contrast FA $>$ CR (false memory; $c$ ) (rendered on a standard brain's left lateral view) increased with age in frontal and parietal regions. Voxel-level threshold, $p<0.001$; cluster extent, $>50$ contiguous voxels. $\boldsymbol{d}$, Overlap of age-related increases of activation for contrasts HIT $>$ CR, HIT $>$ MISS, and FA $>$ CR in left frontal and parietal regions. 
Table 4. Additional retrieval contrasts

\begin{tabular}{|c|c|c|c|c|c|c|}
\hline & \multirow[b]{2}{*}{ BA } & \multicolumn{3}{|c|}{ MNI coordinates } & \multirow{2}{*}{$\begin{array}{l}\text { Peak } t \\
\text { value }\end{array}$} & \multirow{2}{*}{$\begin{array}{l}\text { No. of } \\
\text { voxels }\end{array}$} \\
\hline & & $x$ & $y$ & $z$ & & \\
\hline \multicolumn{7}{|l|}{$F A>C R$ (false memory) across all participants } \\
\hline Left inferior frontal gyrus/insula & $47 / 13$ & -32 & 22 & -4 & 6.11 & 505 \\
\hline Right precuneus & 31 & 20 & -58 & 24 & 5.80 & 983 \\
\hline Right precuneus/superior parietal lobe & 7 & 16 & -68 & 42 & 5.77 & \\
\hline Left precuneus & 31 & -18 & -60 & 24 & 5.72 & 1776 \\
\hline Left precuneus/superior parietal lobe & 7 & -12 & -76 & 46 & 4.81 & \\
\hline \multicolumn{7}{|l|}{ Left/right medial frontal gyrus/ } \\
\hline anterior cingulate & $9 / 8 / 6 / 32$ & -4 & 30 & 34 & 5.56 & 1044 \\
\hline Left middle/inferior frontal gyrus & 10 & -40 & 56 & 6 & 5.54 & 284 \\
\hline Left caudate & n.a. & -10 & 12 & 2 & 5.27 & 174 \\
\hline Right inferior frontal gyrus/insula & $47 / 13 / 45$ & 32 & 26 & -2 & 4.79 & 309 \\
\hline Left inferior/middle frontal gyrus & 9 & -36 & 4 & 30 & 4.60 & 468 \\
\hline Left middle frontal gyrus & 46 & -44 & 26 & 26 & 4.37 & \\
\hline \multicolumn{7}{|l|}{ False memory increased with age } \\
\hline Right posterior cingulate gyrus & $30 / 31$ & 18 & -64 & 12 & 4.57 & 280 \\
\hline Right inferior/middle frontal gyrus & 47 & 30 & 26 & -20 & 5.32 & 295 \\
\hline Left inferior frontal gyrus & 47 & -38 & 28 & 0 & 4.87 & 262 \\
\hline Left cerebellum & n.a. & -14 & -70 & -28 & 4.52 & $122^{b}$ \\
\hline Left caudate & n.a. & -18 & -24 & 26 & 4.26 & $64^{b}$ \\
\hline Left insula/claustrum & 13 & -30 & -12 & 16 & 4.17 & $71^{b}$ \\
\hline Right precuneus & 7 & 12 & -76 & 48 & 3.96 & $71^{b}$ \\
\hline Left superior parietal lobe/precuneus & 7 & -16 & -70 & 58 & 3.88 & $57^{b}$ \\
\hline \multicolumn{7}{|l|}{$\begin{array}{l}\text { CR }>\text { FA (accurate novelty detection) across } \\
\text { all participants }\end{array}$} \\
\hline Right Superior/middle temporal gyrus & $39 / 22 / 37$ & 50 & -64 & 20 & 6.04 & $1038^{a}$ \\
\hline Left an & 32 & -12 & 30 & -4 & 5.87 & 1182 \\
\hline Left $r$ & $10 / 11$ & -4 & 46 & -10 & 5.53 & \\
\hline poral gyrus & $21 / 20$ & -64 & -18 & -10 & 5.76 & 1216 \\
\hline Left $n$ & 38 & -42 & 6 & -44 & 5.33 & \\
\hline mporal gyrus & $39 / 21$ & -44 & -58 & 24 & 4.96 & $786^{a}$ \\
\hline Left infe & 40 & -68 & -34 & 30 & 4.96 & $405^{a}$ \\
\hline Left sup & 22 & -64 & -38 & 12 & 4.47 & \\
\hline poral gyrus & $21 / 22$ & 58 & -4 & -4 & 4.85 & $195^{a}$ \\
\hline pocampal gyrus & $28 / 36 / 35$ & -30 & -4 & -34 & 4.8 & 265 \\
\hline Left hippoc & n.a. & -22 & -12 & -24 & 4.56 & \\
\hline Right/left posterior cingulate & $31 / 23 / 30$ & 2 & -54 & 28 & 4.77 & 454 \\
\hline Left precuneus & 7 & -2 & -58 & 44 & 3.88 & \\
\hline Right parahippocampal gyrus/amygdala & $35 / 34 / 28$ & 22 & -6 & -22 & 4.57 & 264 \\
\hline Right parahippocampal gyrus & 36 & 28 & -4 & -34 & 4.28 & \\
\hline Right putamen & n.a. & 28 & -6 & -8 & 4.51 & 413 \\
\hline Right insula & 13 & 46 & -10 & 14 & 4.14 & \\
\hline Right inferior parietal lobe & 40 & 50 & -34 & 26 & 4.18 & $276^{a}$ \\
\hline Right superior temporal gyrus & 42 & 62 & -28 & 16 & 4.1 & \\
\hline \multicolumn{7}{|l|}{$C R>F A$ increased with age } \\
\hline & & & & & & \\
\hline
\end{tabular}

Regions in bold are also associated with age-related increases in the contrast HIT $>$ CR. n.a., Not applicable. ${ }^{a}$ Regions also showed activations in the contrasts CR $>$ HIT and/or CR $>$ MISS.

${ }^{b}$ Increases with age did not survive FWE cluster-level correction.

ries between two components of memory: (1) a strategic component that specifies the role of cognitive control via frontalparietal networks that undergo protracted maturation and (2) an associative component that specifies the role of binding processes via regions in the MTL and matures early (Shing et al., 2008, 2010). In the present study, there were age-related changes in frontal and parietal regions often associated with strategic control and an absence of age-related changes in MTL regions associated with associative binding.

Although MTL activations associated with successful retrieval did not change with age, there were age-related changes in MTLprefrontal coupling during memory retrieval. With age, there was a change in the successful retrieval connectivity (hits vs correct rejections) of the left and right PHG with the left IFG, a region where activations for successful retrieval increased with age. This finding is similar to a developmental growth of MTL-prefrontal functional connectivity during encoding (Menon et al., 2005) and is consistent with evidence that increased MTL-prefrontal functional connectivity is associated with better memory encoding (Ranganath et al., 2005; Summerfield et al., 2006). An unexpected finding was that the age difference arose from connectivity (temporal correlations) during correct rejections, which did not change with age behaviorally, and that there was no age-related change in connectivity during hits, which did change with age behaviorally and in parietal activation. Perhaps the connectivity differences between MTL and prefrontal cortex reflected a different kind of retrieval operation than that mediated by the parietal cortex. In broad terms, declarative memory for recently formed memories is frequently conceptualized as reflecting an interaction between MTL and neocortical regions, and these findings suggest that this interaction may change with age during successful retrieval.

The identification of brain regions associated with memory retrieval is achieved through convergence of neuropsychology and neuroimaging evidence. Neuropsychological evidence indicates that the MTL is essential for normal recognition memory (Zola-Morgan et al., 1986), although lesion studies cannot dissociate MTL contributions to encoding versus retrieval. Neuroimaging studies, which can identify regions correlated with a function but cannot determine the necessity of those regions for that function, have consistently observed frontal and parietal activation for recognition memory. Here we showed that during memory retrieval, frontal and parietal cortical regions and the caudate undergo protracted functional maturation between midchildhood and adulthood. In addition, there were age related changes in the differential functional connectivity (PPI) between the MTL and frontal cortex during successful memory retrieval (hits vs correct rejections). Future studies will need to characterize what recognition retrieval processes are mediated by frontal and parietal regions and by the basal ganglia and how these may grow in development.

\section{References}

Barnes KA, Cohen AL, Power JD, Nelson SM, Dosenbach YB, Miezin FM, Petersen SE, Schlaggar BL (2010) Identifying basal ganglia divisions in individuals using resting-state functional connectivity MRI. Front Syst Neurosci 4:18.

Buckner RL, Wheeler ME (2001) The cognitive neuroscience of remembering. Nat Rev Neurosci 2:624-634.

Burgund E, Kang HC, Kelly JE, Buckner RL, Snyder AZ, Petersen SE, Schlaggar BL (2002) The feasibility of a common stereotactic space for children and adults in fMRI studies of development. Neuroimage 17:184-200.

Cabeza R (2008) Role of parietal regions in episodic memory retrieval: the dual attentional processes hypothesis. Neuropsychologia 46:1813-1827.

Cabeza R, Ciaramelli E, Olson IR, Moscovitch M (2008) The parietal cortex and episodic memory: an attentional account. Nat Rev Neurosci 9:613-625

Chai XJ, Ofen N, Jacobs LF, Gabrieli JD (2010) Scene complexity: influence on perception, memory, and development in the medial temporal lobe. Front Hum Neurosci 4:21.

Chiu CY, Schmithorst VJ, Brown RD, Holland SK, Dunn S (2006) Making memories: A cross-sectional investigation of episodic memory encoding in childhood using fMRI. Dev Neuropsychol 29:321-340.

Ciaramelli E, Grady CL, Moscovitch M (2008) Top-down and bottomup attention to memory: a hypothesis (AtoM) on the role of the posterior parietal cortex in memory retrieval. Neuropsychologia 46:1828-1851.

Ciaramelli E, Grady C, Levine B, Ween J, Moscovitch M (2010) Top-down and bottom-up attention to memory are dissociated in posterior parietal cortex: neuroimagingand and neuropsychological evidence. J Neurosci 30:4943-4956. 
Dobbins IG, Han S (2006) Cue- versus probe-dependent prefrontal cortex activity during contextual remembering. J Cogn Neurosci 18:1439-1452.

Dobbins IG, Foley H, Schacter DL, Wagner AD (2002) Executive control during episodic retrieval: multiple prefrontal processes subserve source memory. Neuron 35:989-996.

Eickhoff SB, Stephan KE, Mohlberg H, Grefkes C, Fink GR, Amunts K, Zilles K (2005) A new SPM toolbox for combining probabilistic cytoarchitectonic maps and functional imaging data. Neuroimage 25:1325-1335.

Fletcher PC, Shallice T, Frith CD, Frackowiak RS, Dolan RJ (1998) The functional roles of prefrontal cortex in episodic memory. II. Retrieval. Brain 121:1249-1256.

Friston KJ, Buechel C, Fink GR, Morris J, Rolls E, Dolan RJ (1997) Psychophysiological and modulatory interactions in neuroimaging. Neuroimage 6:218-229.

Ghetti S, DeMaster DM, Yonelinas AP, Bunge SA (2010) Developmental differences in medial temporal lobe function during memory encoding. J Neurosci 30:9548-9556.

Giedd JN, Blumenthal J, Jeffries NO, Castellanos FX, Liu H, Zijdenbos A, Paus T, Evans AC, Rapoport JL (1999) Brain development during childhood and adolescence: a longitudinal MRI study. Nat Neurosci 2:861-863.

Gogtay N, Giedd JN, Lusk L, Hayashi KM, Greenstein D, Vaituzis AC, Nugent TF 3rd, Herman DH, Clasen LS, Toga AW, Rapoport JL, Thompson PM 2004 Dynamic mapping of human cortical development during childhood through early adulthood. Proc Natl Acad Sci U S A 101, 8174-8179.

Gogtay N, Nugent TF 3rd, Herman DH, Ordonez A, Greenstein D, Hayashi KM, Clasen L, Toga AW, Giedd JN, Rapoport JL, Thompson PM 2006 . Dynamic mapping of normal human hippocampal development. Hippocampus $16,664-672$.

Haramati S, Soroker N, Dudai Y, Levy DA (2008) The posterior parietal cortex in recognition memory: a neuropsychological study. Neuropsychologia 46:1756-1766.

Hutchinson JB, Uncapher MR, Wagner AD (2009) Posterior parietal cortex and episodic retrieval: convergent and divergent effects of attention and memory. Learn Mem 16:343-356.

Kahn I, Davachi L, Wagner AD (2004) Functional-neuroanatomic correlates of recollection: implications for models of recognition memory. J Neurosci 24:4172-4180.

Kang HC, Burgund ED, Lugar HM, Petersen SE, Schlaggar BL (2003) Comparison of functional activation foci in children and adults using a common stereotactic space. Neuroimage 19:16-28.

Konishi S, Wheeler ME, Donaldson DI, Buckner RL (2000) Neural correlates of episodic retrieval success. Neuroimage 12:276-286.

Maril A, Davis PE, Koo JJ, Reggev N, Zuckerman M, Ehrenfeld L, Mulkern RV, Waber DP, Rivkin MJ (2010) Developmental fMRI study of episodic verbal memory encoding in children. Neurology 75:2110-2116.

Menon V, Boyett-Anderson JM, Reiss AL (2005) Maturation of medial temporal lobe response and connectivity during memory encoding. Brain Res Cogn Brain Res 25:379-385.

Montaldi D, Spencer TJ, Roberts N, Mayes AR (2006) The neural system that mediates familiarity memory. Hippocampus 16:504-520.

O'Connor AR, Han S, Dobbins IG (2010) The inferior parietal lobule and recognition memory: expectancy violation or successful retrieval? J Neurosci 30:2924-2934.

Ofen N, Kao YC, Sokol-Hessner P, Kim H, Whitfield-Gabrieli S, Gabrieli JD (2007) Development of the declarative memory system in the human brain. Nat Neurosci 10:1198-1205.

Passingham D, Sakai K (2004) The prefrontal cortex and working memory: physiology and brain imaging. Curr Opin Neurobiol 14:163-168.
Paz-Alonso PM, Ghetti S, Donohue SE, Goodman GS, Bunge SA (2008) Neurodevelopmental correlates of true and false recognition. Cereb Cortex 18:2208-2216.

Petrides M, Alivisatos B, Evans AC, Meyer E (1993) Dissociation of human mid-dorsolateral from posterior dorsolateral frontal cortex in memory processing. Proc Natl Acad Sci U S A 90:873-877.

Ranganath C, Heller A, Cohen MX, Brozinsky CJ, Rissman J (2005) Functional connectivity with the hippocampus during successful memory formation. Hippocampus 15:997-1005.

Roediger HL 3rd, Watson JM, McDermott KB, Gallo DA (2001) Factors that determine false recall: a multiple regression analysis. Psychon Bull Rev 8:385-407.

Rugg MD, Fletcher PC, Chua PM, Dolan RJ (1999) The role of the prefrontal cortex in recognition memory and memory for source: an fMRI study. Neuroimage 10:520-529.

Sestieri C, Shulman GL, Corbetta M (2010) Attention to memory and the environment: functional specialization and dynamic competition in human posterior parietal cortex. J Neurosci 30:8445-8456.

Shing YL, Werkle-Bergner M, Li SC, Lindenberger U (2008) Associative and strategic components of episodic memory: a life-span dissociation. J Exp Psychol Gen 137:495-513.

Shing YL, Werkle-Bergner M, Brehmer Y, Müller V, Li SC, Lindenberger U (2010) Episodic memory across the lifespan: the contributions of associative and strategic components. Neurosci Biobehav Rev 34:1080-1091.

Sowell ER, Peterson BS, Thompson PM, Welcome SE, Henkenius AL, Toga AW (2003) Mapping cortical change across the human life span. Nat Neurosci 6:309-315.

Sowell ER, Thompson PM, Leonard CM, Welcome SE, Kan E, Toga AW (2004) Longitudinal mapping of cortical thickness and brain growth in normal children. J Neurosci 24:8223-8231.

Spaniol J, Davidson PS, Kim AS, Han H, Moscovitch M, Grady CL (2009) Event-related fMRI studies of episodic encoding and retrieval: metaanalyses using activation likelihood estimation. Neuropsychologia 47:1765-1779.

Summerfield C, Greene M, Wager T, Egner T, Hirsch J, Mangels J (2006) Neocortical connectivity during episodic memory formation. PLoS Biol 4:e128.

Thompson-Schill SL (2003) Neuroimaging studies of semantic memory: inferring "how" from "where." Neuropsychologia 41:280-292.

Thompson-Schill SL, D’Esposito M, Aguirre GK, Farah MJ (1997) Role of left inferior prefrontal cortex in retrieval of semantic knowledge: a reevaluation. Proc Natl Acad Sci U S A 94:14792-14797.

Vilberg KL, Rugg MD (2008) Memory retrieval and the parietal cortex: a review of evidence from a dual-process perspective. Neuropsychologia 46:1787-1799.

Wagner AD, Paré-Blagoev EJ, Clark J, Poldrack RA (2001) Recovering meaning: left prefrontal cortex guides controlled semantic retrieval. Neuron 31:329-338.

Wagner AD, Shannon BJ, Kahn I, Buckner RL (2005) Parietal lobe contributions to episodic memory retrieval. Trends Cogn Sci 9:445-453.

Wheeler ME, Buckner RL (2003) Functional dissociation among components of remembering: Control, perceived oldness, and content. J Neurosci 23:3869-3880.

Yonelinas AP, Otten LJ, Shaw KN, Rugg MD (2005) Separating the brain regions involved in recollection and familiarity in recognition memory. J Neurosci 25:3002-3008.

Zola-Morgan S, Squire LR, Amaral DG (1986) Human amnesia and the medial temporal region: enduring memory impairment following a bilateral lesion limited to field CA1 of the hippocampus. J Neurosci 6:2950 2967. 